Revista de Matemática: Teoría y Aplicaciones 2005 12(1 \& 2) : 45-50

CIMPA - UCR - CCSS ISSN: 1409-2433

\title{
¿COMO TRABAJAN LAS CALCULADORAS?
}

\author{
BruCE H. EDWARDS*
}

Received/Recibido: 24 Feb 2004

\begin{abstract}
What happens when you press the sine key on your calculator? How does a calculator quickly produce the values of trigonometric functions? For instance, how does the TI-89 calculate sin 1.0? You might be surprised to know that the calculator does not use series or polynomial approximation, but rather a simple algorithm called the CORDIC method. In fact, the CORDIC method is used for almost all function evaluations (trigonometric, logarithmic, exponential, hyperbolic, inverse functions, etc.), and even multiplication and division. In this paper we will present the main ideas of the CORDIC method along with some elementary examples.
\end{abstract}

Keywords: Graphic calculator, CORDIC.

\section{Resumen}

¿Qué ocurre cuando un estudiante presiona la tecla de la función tangente en su calculadora? ¿Cómo hace una calculadora para producir rápidamente los valores de las funciones trigonométricas? Por ejemplo: ¿Cómo hace la TI-89 para calcular tan(1.0)? Es sorprendente saber que la calculadora no utiliza series de Taylor, ni aproximaciones polinómicas, sino que usa un simple algoritmo llamado CORDIC. El método de CORDIC es el que la calculadora usa para evaluar la mayoría de las funciones (trigonométricas, logarítmicas, exponenciales, hiperbólicas, funciones inversas, etc.), y aún más, se usa el mismo método para efectuar multiplicaciones y divisiones. En nuestra charla se presentarán las principales ideas del método de CORDIC, junto con algunos ejemplos elementales.

Palabras clave: Calculadora gráfica, CORDIC.

Mathematics Subject Classification: 65Y99.

*Department of Mathematics, 358 Little Hall, University of Florida, Gainesville, FL 32611-8105, U.S.A. E-Mail: be@math.ufl.edu 


\section{Introduction}

The CORDIC method for approximating function values is used on all popular graphing calculators, including the TI-81, TI-82, TI-85 and HP-48G. This algorithm is not based on calculus nor polynomial approximation, but instead involves a more elementary system of iterative equations. The CORDIC (COordinate Rotation DIigital Computer) algorithm is relatively new, introduced in 1959 by Volder [4] to calculate trigonometric function values. It was later extended to hyperbolic, logarithmic, and other functions by Walther in 1971 [5].

The hardware restrictions of calculators require that the elementary functions be computed using only additions, subtractions, digit shifts, comparisons, and the recall of a small number of stored constants. As we will see, the CORDIC method only uses these five basic operations. It is interesting to note that even multiplication and division can be computed by the CORDIC method.

Because it is time-consuming to convert from base 10 to base 2, the CORDIC algorithm is programmed in base 10. However, it is easier to understand the essential ideas using binary arithmetic. Hence, multiplication by $2^{k}$, where $k$ is an integer, is nothing more than a digit shift. See the UMAP module [1] for an elementary treatment of the CORDIC method using base 10 arithmetic.

We will not address in full detail the important theoretical issue of the convergence properties of the CORDIC method, and the interested reader is referred to the literature [2]. In general, the algorithm consists of performing $n$ iterations of the three equations given below, and the convergence theorems guarantee that the resulting answer is within a certain error bound that depends on $n$.

\section{The CORDIC Algorithm}

The general CORDIC algorithm consists of the following three iterative equations.

$$
\begin{aligned}
x_{k+1} & =x_{k}-m \delta_{k} y_{k} 2^{-k} \\
y_{k+1} & =y_{k}+\delta_{k} x_{k} 2^{-k} \\
z_{k+1} & =z_{k}-\delta_{k} \sigma_{k}
\end{aligned}
$$

The constants $m, \delta_{k}$, and $\sigma_{k}$ depend on the specific computation being performed, as explained below.

1. $m$ is either 0,1 , or $-1 . m=1$ is used for trigonometric and inverse trigonometric functions. $m=-1$ is used for hyperbolic, inverse hyperbolic, exponential, and logarithmic functions, as well as square roots. Finally, $m=0$ is used for multiplication and division.

2. $\delta_{k}$ is one of the following two signum functions:

$$
\delta_{k}=\operatorname{sgn}\left(z_{k}\right)=\left\{\begin{array}{ll}
1 & z_{k} \geq 0 \\
-1 & z_{k}<0
\end{array} \quad \text { or } \quad \delta_{k}=-\operatorname{sgn}\left(y_{k}\right)= \begin{cases}1, & y_{k}<0 \\
-1, & y_{k} \geq 0\end{cases}\right.
$$


The first is often called the rotation mode, in which the $z$ values are driven to zero, whereas the second is the vectoring mode, in which the $y$ values are driven to zero. Note that $\delta_{k}$ requires nothing more than a comparison.

3. The numbers $\sigma_{k}$ are stored constants which depend on the value of $m$. For $m=1$, $\sigma_{k}=\tan ^{-1} 2^{-k}$, for $m=0, \sigma_{k}=2^{-k}$, and for $m=-1, \sigma_{k}=\tanh ^{-1} 2^{-k}$ (with some minor modifications to be discussed later).

To use these equations, appropriate starting values $x_{0}, y_{0}$, and $z_{0}$ must be given. One of these inputs, say $z_{0}$, might be the number whose sine we wish to approximate, $\sin z_{0}$. Or two of these inputs, say $y_{0}$ and $x_{0}$, might be the quotient we wish to approximate, $y_{0} / x_{0}$. In all cases, the starting values must be restricted to a certain interval about the origin in order to ensure convergence [5]. As we shall see in the following two examples, one of the variables tends to zero while another variable approaches the desired approximation.

\section{Example: Division}

Let $m=0, \delta_{k}=-\operatorname{sgn}\left(y_{k}\right)$, and $\sigma_{k}=2^{-k}$. Then the following equations will approximate the quotient $y_{0} / x_{0}$ for $\left|y_{0} / x_{0}\right| \leq 2$. Notice that these equations consist of only additions, subtractions, comparisons and binary shifts.

$$
\begin{aligned}
& x_{k+1}=x_{0} \\
& y_{k+1}=y_{k}+\delta_{k} x_{0} 2^{-k} \\
& z_{k+1}=z_{k}-\delta_{k} 2^{-k} \quad\left(\sigma_{k}=2^{-k}\right)
\end{aligned}
$$

The starting values are the given numbers $x_{0}, y_{0}$ and $z_{0}=0$. Here are the first five and last five iterations for the specific example $x_{0}=5$ and $y_{0}=7$ ( $n=50$ iterations):

$\begin{array}{cccc} & x & y & z \\ 0 & 5.00000000000000 & 7.00000000000000 & 0 \\ 1 & 5.00000000000000 & 2.00000000000000 & 1.00000000000000 \\ 2 & 5.00000000000000 & -0.50000000000000 & 1.50000000000000 \\ 3 & 5.00000000000000 & 0.75000000000000 & 1.25000000000000 \\ 4 & 5.00000000000000 & 0.12500000000000 & 1.37500000000000 \\ 5 & 5.00000000000000 & -0.18750000000000 & 1.43750000000000 \\ \vdots & & & \\ 46 & 5.00000000000000 & -0.00000000000003 & 1.40000000000001 \\ 47 & 5.00000000000000 & 0.00000000000004 & 1.39999999999999 \\ 48 & 5.00000000000000 & 0.00000000000001 & 1.40000000000000 \\ 49 & 5.00000000000000 & -0.00000000000001 & 1.40000000000000 \\ 50 & 5.00000000000000 & -0.00000000000000 & 1.40000000000000\end{array}$

Notice how the $y$ values have been driven towards zero while the $z$ values approximate the quotient $y_{0} / x_{0}=1.4$. 
It is not difficult to see why the sequence $z_{k}$ will approach $y_{0} / x_{0}$. Note that the second and third equations imply that

$$
y_{n+1}=y_{0}+\sum_{k=0}^{n} \delta_{k} x_{0} 2^{-k} \text { and } z_{n+1}=-\sum_{k=0}^{n} \delta_{k} 2^{-k},
$$

respectively. Hence,

$$
\frac{y_{0}}{x_{0}}=\frac{y_{n+1}-\sum_{k=0}^{n} \delta_{k} x_{0} 2^{-k}}{x_{0}}=\frac{y_{n+1}}{x_{0}}-\sum_{k=0}^{n} \delta_{k} 2^{-k}=\frac{y_{n+1}}{x_{0}}+z_{n+1},
$$

which gives

$$
\left|z_{n+1}-\frac{y_{0}}{x_{0}}\right|=\left|\frac{y_{n+1}}{x_{0}}\right| .
$$

Since $y_{n+1}$ is close to zero, $z_{n+1} \approx y_{0} / x_{0}$.

\section{Example: Sine and Cosine}

To approximate the sine and cosine of a number $z_{0}=\theta,-\pi / 2 \leq \theta \leq \pi / 2$, we use $m=1$, $\delta_{k}=\operatorname{sgn}\left(z_{k}\right)$, and $\sigma_{k}=\tan ^{-1} 2^{-k}$.

$$
\begin{aligned}
& x_{k+1}=x_{k}-\delta_{k} y_{k} 2^{-k} \\
& y_{k+1}=y_{k}+\delta_{k} x_{k} 2^{-k} \\
& z_{k+1}=z_{k}-\delta_{k} \tan ^{-1} 2^{-k} \quad\left(\sigma_{k}=\tan ^{-1} 2^{-k}\right)
\end{aligned}
$$

The starting values are $x_{0}=K=\prod_{j=0}^{n} \cos \left(\sigma_{j}\right), y_{0}=0$, and $z_{0}=\theta$, the given angle. Because of the way the $z$ 's are constructed, $z_{k}$ is forced towards zero. As we see in the following example, the $x$ 's will tend to $\cos \theta$ and the $y$ 's to $\sin \theta$. A precise proof of convergence depends on elementary trigonometric identities and can be found in [2].

The following MATLAB program does $n=47$ iterations of the CORDIC algorithm to approximate the sine and cosine of the angle $\theta,-\pi / 2 \leq \theta \leq \pi / 2$. We show the output for the first five and last five iterations for the calculation of the sine and cosine of $\theta=1$. Notice how the $z$ values are being driven to zero, while the $x$ values approach $\cos 1$ and the $y$ values approach $\sin 1$.

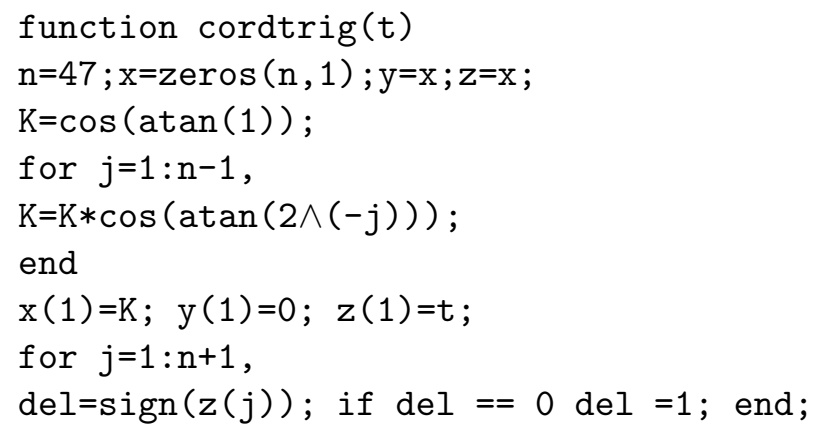




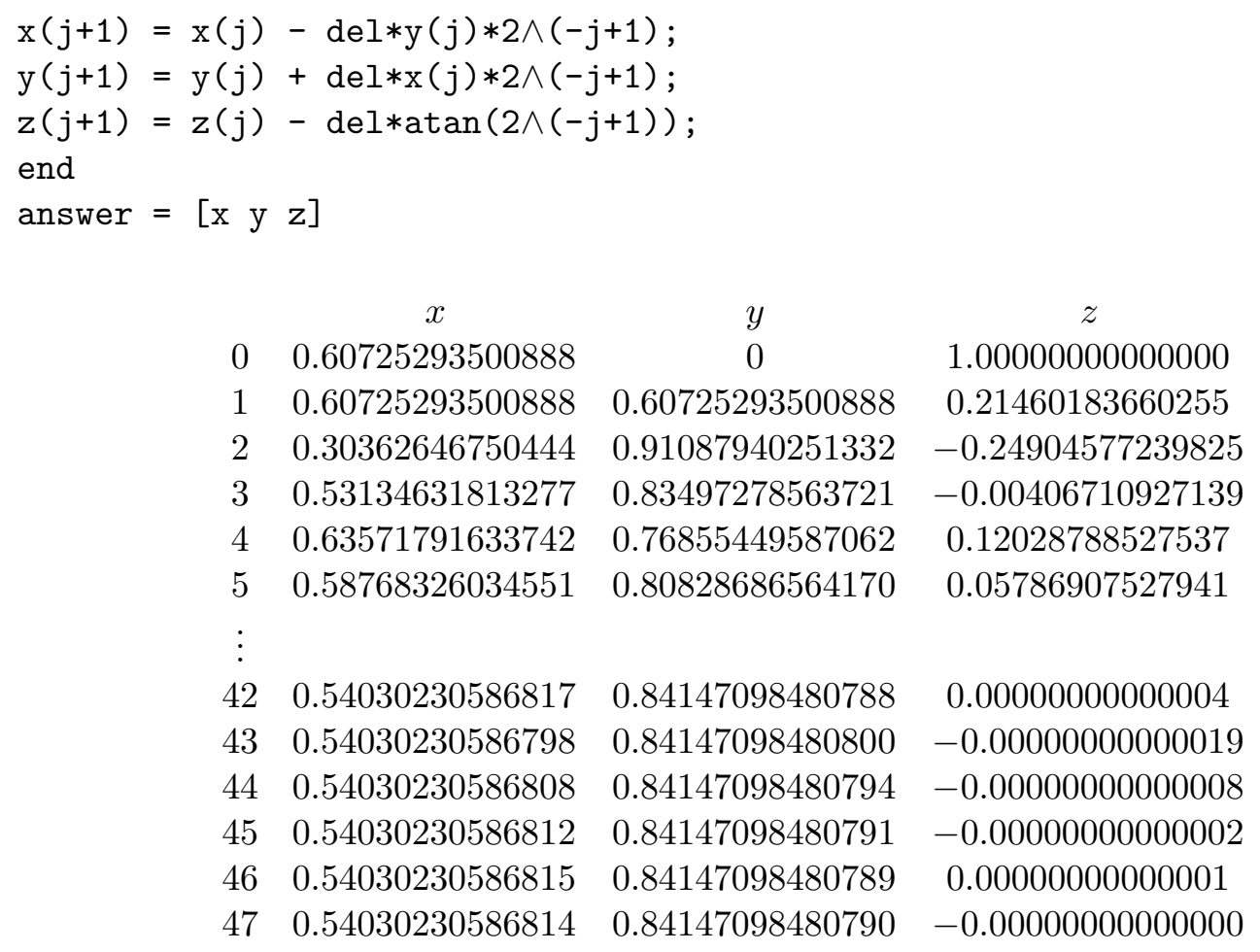

$\begin{array}{cccc} & x & y & z \\ 0 & 0.60725293500888 & 0 & 1.00000000000000 \\ 1 & 0.60725293500888 & 0.60725293500888 & 0.21460183660255 \\ 2 & 0.30362646750444 & 0.91087940251332 & -0.24904577239825 \\ 3 & 0.53134631813277 & 0.83497278563721 & -0.00406710927139 \\ 4 & 0.63571791633742 & 0.76855449587062 & 0.12028788527537 \\ 5 & 0.58768326034551 & 0.80828686564170 & 0.05786907527941 \\ \vdots & & & \\ 42 & 0.54030230586817 & 0.84147098480788 & 0.00000000000004 \\ 43 & 0.54030230586798 & 0.84147098480800 & -0.00000000000019 \\ 44 & 0.54030230586808 & 0.84147098480794 & -0.00000000000008 \\ 45 & 0.54030230586812 & 0.84147098480791 & -0.00000000000002 \\ 46 & 0.54030230586815 & 0.84147098480789 & 0.00000000000001 \\ 47 & 0.54030230586814 & 0.84147098480790 & -0.00000000000000\end{array}$

\section{Concluding Remarks}

The above examples show some of the versatility of the CORDIC method. In the following table we give a more complete summary of the CORDIC options.

$$
\begin{array}{lll}
\delta_{k}=\operatorname{sgn}\left(z_{k}\right) \quad\left(z_{k} \rightarrow 0\right) & & \\
\delta_{k}=-\operatorname{sgn}\left(y_{k}\right) \quad\left(y_{k} \rightarrow 0\right) & & \\
m=0 & x_{0}, z_{0} \text { given } y_{0}=0 & x_{0}, y_{0} \text { given } z_{0}=0 \\
\sigma_{k}=2^{-k} & \text { gives } y_{n} \approx x_{0} z_{0} & \text { gives } z_{n} \approx y_{0} / x_{0} \\
m=1 & x_{0}=K, z_{0}=\theta, y_{0}=0 & x_{0}, y_{0} \operatorname{given} z_{0}=0 \\
\sigma_{k}=\tan ^{-1} 2^{-k} & \text { gives } x_{n} \approx \cos \theta, y_{n} \approx \sin \theta & z_{n} \approx \tan ^{-1}\left(y_{0} / x_{0}\right) \\
m=-1 & x_{0}=K^{\prime}, z_{0}=\theta, y_{0}=0 & x_{0}, y_{0} \operatorname{given}_{0}=0 \\
\sigma_{k}=\tanh ^{-1} 2^{-k} & \text { gives } x_{n} \approx \cosh \theta, y_{n} \approx \sinh \theta & z_{n} \approx \tanh ^{-1}\left(y_{0} / x_{0}\right) \\
\text { (some } \sigma_{k} \text { repeated) } & e^{\theta} \approx x_{n}+y_{n} & x_{n} \approx \sqrt{x_{0}^{2}-y_{0}^{2}} / K^{\prime}
\end{array}
$$

In this table, $K=\prod_{j=0}^{n} \cos \left(\sigma_{j}\right)$ and $K^{\prime}$ is a similar product using the hyperbolic cosine function. It should be noted that the key convergence theorem [2] for the CORDIC algorithm requires that the constants $\sigma_{k}$ satisfy the inequality

$$
\sigma_{k} \leq \sum_{j=k+1}^{n} \sigma_{j}+\sigma_{n}, \quad \text { for } \quad 0 \leq k \leq n .
$$


It is an easy exercise to show that the constants $\sigma_{k}=2^{-k}$ and $\sigma_{k}=\tan ^{-1} 2^{-k}$ satisfy this property. However, for the hyperbolic functions, $m=-1$, the constants $\sigma_{k}=\tanh ^{-1} 2^{-k}$ do not satisfy this property for all $k$. Hence, it is necessary to repeat certain $\sigma_{k}$ values, the details of which can be found in [5].

To calculate $\ln w$, you can use the identity $\tanh ^{-1} t=\frac{1}{2} \ln \frac{1+t}{1-t}$ and the CORDIC hyperbolic case, starting with $x_{0}=w+1, y_{0}=w-1$, to obtain

$$
z_{n} \approx \tanh ^{-1}\left(y_{0} / x_{0}\right)=\frac{1}{2} \ln \left(\frac{1+y_{0} / x_{0}}{1-y_{0} / x_{0}}\right)=\frac{1}{2} \ln \left(\frac{x_{0}+y_{0}}{x_{0}-y_{0}}\right)=\frac{1}{2} \ln w .
$$

Thus, $\ln w \approx 2 z_{n}$. Finally, it can be shown that using $x_{0}=w+0.25$ and $y_{0}=w-0.25$ you obtain $\sqrt{w} \approx K^{\prime} x_{n}$.

\section{References}

[1] Pulskamp, R.J.; Delaney, J.A. (1991) "Computer and Calculator Computation of Elementary Functions", UMAP Module 708.

[2] Schelin, C.W. (1983) "Calculator function approximation", American Mathematical Monthly 90(5): 317-325.

[3] "Transcendental Function Algorithms", Post from Texas Instruments to Graph-TI mailing list, March 8, 1993.

[4] Volder, J.E. (1959) "The CORDIC trigonometric computing technique", IRE Transactions on Electronic Computers, volume EC-8, No. 3, September: 330-334.

[5] Walther, J.S. (1971) "A unified algorithm for elementary functions", Joint Computer Conference Proceedings, Spring: 379-385. 